\title{
Relative merits of using numbers and biomass in fish community studies
}

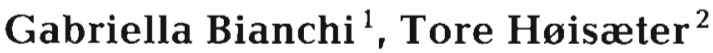 \\ ${ }^{1}$ Institute of Marine Research, Division for International Development Programmes, PO Box 1870, Nordnes, N-5024 Bergen, Norway \\ ${ }^{2}$ Department of Fisheries and Marine Biology, University of Bergen, Høyteknologisenteret, N-5020 Bergen, Norway
}

\begin{abstract}
In descriptions of fish communities, the question of which measure of abundance should be used, whether numbers or biomass, has never been addressed. While both measures are often available, the common practice is to use numerical abundance for such descriptions, without any explicit justification for this choice. In order to contribute to the clarification of this issue, we have compared correspondence analyses/TWIA-classifications performed on both the numerical density and the biomass of the same trawl catches from a region off the western coast of Africa. The situation in which the quantitative aspect of the samples is disregarded, represented by presence/absence analyses, is illustrated for comparison. As it appeared likely that the length of the ecological or biogeographical 'gradient' would be of importance in how different the results of the 2 abundance measures would emerge, a series of subsamples of the total material, representing shorter ecological gradients, was also analysed. The analyses show that in most situations the choice between numbers and biomass matters little. However, in the case of short ecological 'gradients', when all or the large majority of species are common to all samples studied, and the individual fish sizes are different, there may be a marked difference in the patterns shown by the alternative measures of abundance.
\end{abstract}

\section{INTRODUCTION}

In the quantitative description of animal communities, numerical abundance is most commonly used as abundance measure. This trend is also reflected in the study of fish communities (Day \& Pearcy 1968, Markle \& Musick 1974, Haedrich et al. 1975, Chavez 1979, Haedrich et al. 1980, Yañéz-Arancibia et al. 1980 , Colvocoresses \& Musick 1984, Costa 1986, Bennett 1989, Mahon \& Smith 1989, Mas-Riera et al. 1990, Bergstad 1991), with the exception of a few, fisheryrelated studies, where it seemed more natural to use biomass values (Gabriel \& Tyler 1980, Overholtz \& Tyler 1984, McManus 1985, Bianchi 1991). Presence/ absence has also been used by some authors (Fager \& Longhurst 1968, Warburton 1978, Vargas Maldonado et al. 1981, Lleonart \& Roel 1984, Rainer 1984, YañézArancibia et al. 1985, Roel 1987).

While a number of authors have discussed which value to use in the description of community structure, for example diversity measures (e.g. Wilhm 1968 and Bechtel \& Copeland 1970), this question does not appear to have been seriously addressed for multivariate analytical techniques used to identify species associations in a given sampling area. In the present study we confine ourselves to considerations of the use of numerical abundance vs biomass for multivariate analyses to detect patterns of species associations by, in particular, (Detrended) Correspondence Analysis [(D)CA; Hill \& Gauch 1980] and Two-Way Indicator species Analysis (TWIA; Hill 1979).

\section{MATERIALS AND METHODS}

Materials. The data used in the present study are taken from the investigation on the demersal assemblages off Congo and Gabon (Bianchi 1992b). The data were collected in January-February 1989, and comprise a total of 94 stations and 314 species. The geographical area included, and the equipment used, are described in Bianchi (1992b). Both numbers and biomass were recorded for each species caught in this survey, but only the biomass values were used in the 
analysis of the demersal assemblages. In the present article, analyses based on the numerical abundances are compared with equivalent analyses based on biomass values. Presence/absence values are included as well, whenever it was felt appropriate. As there is reason to believe that the lengths of the environmental gradients covered are of importance for interpreting the results, several data sets representing ecological gradients of different lengths are included in the study. The main ecological gradient is represented by the first axis of Detrended Correspondence Analysis, its length expressed in standard deviation units (SDU) (Jongman et al. 1987). Thus, in addition to the complete material (as presented in Bianchi 1992b) representing the longest gradient (ca 9.4 SDU), successive subsets of the total are used to represent shorter gradients. Thus a medium gradient (5.4 SDU) is represented by a subset of 75 stations, and 3 short gradients $(2.6,2.3$, and 1.7 SDU) consist of 24,21 , and 16 stations respectively.

Methods. Following the methods adopted in a series of papers by the first author on the benthic assemblages outside the coast of several tropical areas, viz. the Pacific coast of Central America (Bianchi 1991), Angola (Bianchi 1992a) and Congo-Gabon (Bianchi 1992b), DCA and TWIA have been singled out for detailed analysis. These techniques have been in common use among community ecologists for several years (e.g. Jongman et al. 1987), and should need no further introduction. The computer programs for the implementation of DCA and other ordination techniques are all included in the program package CANOCO (ter Braak 1990). While a certain element of arbitrariness is introduced in DCA through the detrending procedure (see Jackson \& Somers 1991), this is probably of no concern as long as the same method is used throughout. In the present case, the default value (26) of number of segments in the detrending procedure is used.

TWIA (implemented by the program TWINSPAN; Hill 1979) produces a hierarchical classification in which the number of groups is doubled at each division level. Thus at division level 1, 2 groups are generated, 4 groups at level 2, and so on. TWIA is related to (D)CA in the sense that the ranking of the stations is based on the first (D)CA-axis. However, the ways the abundances are used in these 2 programs are different. While abundance values are used as input to DCA, in TWIA, the abundance values are replaced by so-called pseudospecies. The pseudospecies cut levels for numerical abundance were set to reflect the range of abundances in the data set 10 , $10,100,1000$, and 10000). The cut levels for biomass were chosen so that a rough correspondence to the categories used for numerical abundance was estab- lished, thus facilitating comparison between the results. The mean weight for all fishes caught was calculated, and cut levels roughly corresponding to $10,100,1000$, and 10000 such 'average' fishes were selected. The resulting cut levels were then rounded to $0,0.5,5,50$, and $500 \mathrm{~kg}$.

Presence/absence was used as an abundance measure' in 2 ways: to serve as a comparison with numerical abundance and biomass for the data sets representing the 3 longest gradients in the DCA, and, with the help of TWINSPAN, to define 'independent' groups of reference stations for all data sets. For the 2 longest gradients, the number of groups thus defined was 8 , while the number of groups varied between 4 and 7 for the 3 shortest gradients. The TWINSPAN groups defined in this way were used to identify and circumscribe faunally related stations in the various DCA plots, with the 4 groups generated at level 2 of TWINSPAN as main groups, and the extra groups generated at level 3 as subgroups.

Both numerical and biomass values were $\ln (x+1)$ transformed before analysis with DCA. This is common practice in community studies to reduce the influence of aberrant high values, to give less weight to dominant species, and thus to increase the weight given to the qualitative aspect of the data.

The results from the DCA analyses based on numerical abundance and biomass (DCAn and DCAb) were compared by RDA (Redundancy Analysis), the canonical form of PCA (Principal Component Analysis). The Pearson product-moment-, and Spearman rank correlation coefficients between the station scores on each of the 4 axes obtained from DCAn and DCAb are also presented.

The station scores from DCAn and DCAb were used as analogues of species abundances and environmental variables, respectively, in the input to RDA. To test the hypothesis that the results from the 2 ordinations are completely independent, a Monte Carlo permutation test was performed, using the residuals after fitting 'environmental' (i.e. DCAb) variables (ter Braak 1990). As this hypothesis was rejected for all our data sets, and the F-type test criterion was considered unsuitable for comparing the results across different gradient lengths, the sum of all canonical eigenvalues (trace) was used as a measure of how well the 2 sets agreed. As the sum of all unconstrained eigenvalues in PCA/RDA is always equal to 1 (because of the scaling of the species data; ter Braak 1990), in this case the sum of the canonical eigenvalues is equal to the ratio between the 2 sets.

The results from TWINSPAN were compared by calculating the proportions of stations common to 
corresponding clusters at each level of classification. This was done with a variant of the Jaccard index of similarity:

$$
S_{\jmath}=\frac{\sum_{i=1}^{m_{j}} c_{i}}{\sum_{i=1}^{m_{j}}\left(a_{i}+b_{i}+c_{i}\right)}
$$

where $S_{j}$ expresses the similarity between the classifications derived from numerical and biomass abundances, for division level $j_{i} m_{j}=$ the number of clusters at division level $j_{;} c_{i}=$ the number of shared elements of the $i$ th cluster; $a_{i}$ and $b_{i}=$ the elements unique to the ith cluster of each classification. $S_{j}$ varies between 0 (no element in common in any of the clusters) and 1 (all elements in corresponding clusters are shared).

\section{RESULTS}

\section{Long gradient}

Fig. 1 shows all 94 Congo-Gabon stations in a plane defined by the first 2 DCA-axes, based on numerical abundance and biomass. Table 1 shows the eigenvalues and the gradient lengths for both of these abundance measures, as well as for presence/absence data. The first axis of this data set covers a gradient of about 9

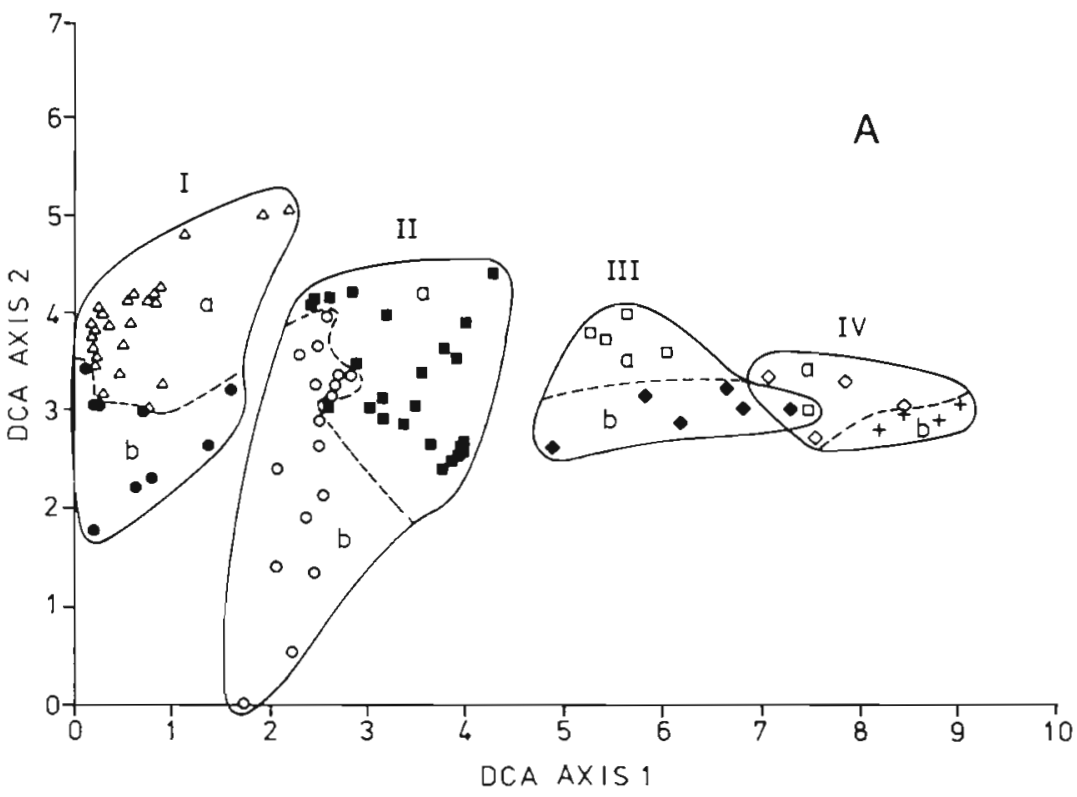

Fig. 1. Detrended correspondence analysis of the 94 stations of the Congo-Gabon material, long gradient (SDU, standard deviation units). Plots based on (A) numerical abundance and (B) biomass. Corresponding groups from TWINSPAN based on presence/absence data are indicated by different symbols, Roman numerals and lower-case letters

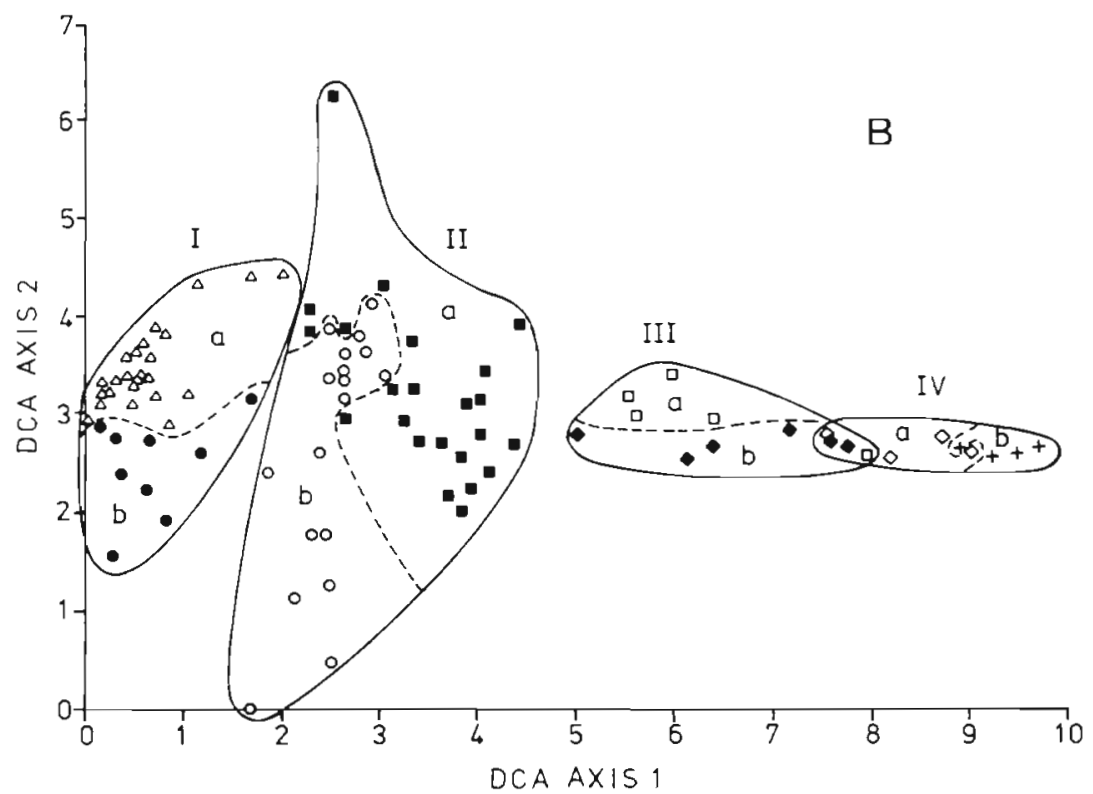


Table 1. Eigenvalues and lengths of environmental gradient (SDU, standard deviation units) for the first 4 axes of DCAn, DCAb and DCAp/a, long gradient (34 stations)

\begin{tabular}{|lcccc|}
\hline & Axis 1 & Axis 2 & Axis 3 & Axis 4 \\
\hline Eigenvalue & & & & \\
DCAn & 0.883 & 0.415 & 0.335 & 0.256 \\
DCAb & 0.907 & 0.467 & 0.381 & 0.285 \\
DCAp/a & 0.868 & 0.424 & 0.326 & 0.247 \\
Length of gradient & & & \\
DCAn & 8.7 & 4.9 & 3.3 & 3.3 \\
DCAb & 9.4 & 6.0 & 3.2 & 5.0 \\
DCAp/a & 8.6 & 5.0 & 3.2 & 4.0 \\
\hline
\end{tabular}

SDU and the stations are arranged from left to right according to increasing depth (from about 10 to $750 \mathrm{~m}$ ). The similarity between the 2 plots is striking and the various station groups lie in similar positions and with approximately the same degree of overlap between groups. However, some minor differences in the position of a few stations are apparent, especially along the second axis. In particular, the uppermost station (in Subgroup IIa) in the biomass plot appears as an outlier. This is due to the presence of some rare species, combined with relatively low biomass for the common species. These common species still dominate in terms of numerical abundance, however, so that this particular station does not deviate from the majority of the stations in this subgroup in the DCAn plot. The apparent similarity between the 2 ordinations is confirmed by the high value of the trace $(0.927$; Table 2$)$, as well as a very high $F$-ratio. The correlation (Table 2) between Axis 1 in the DCAn and DCAb is very high, and still rather high for Axis 2. For the lower axes the correlation is low or negative, and probably not significantly different from zero. The general impression is that the groups appear somewhat better defined and perhaps with less overlap in the DCAn than in the DCAb plot.

As seen in Table 3, the correspondence is absolute between the 2 TWINSPAN classifications for level 1 , and still very high for level 2 . For levels 3 and 4 the correspondence is still acceptable, while there is a sharp decline for level 5 .
Table 2. Summary of the various correlations between scores from the DCA based on numerical abundance and biomass

\begin{tabular}{|c|c|c|c|c|}
\hline & \multirow[t]{2}{*}{ Pearson } & \multirow[t]{2}{*}{ Spearman } & \multicolumn{2}{|c|}{$\mathrm{RDA}$} \\
\hline & & & $\begin{array}{l}\text { Overall } \\
F \text {-ratio }\end{array}$ & Trace \\
\hline \multicolumn{5}{|c|}{ Long gradient $(8.7,9.4$ SDU) } \\
\hline Axis 1 & 0.997 & 0.992 & 281.14 & 0.927 \\
\hline Axis 2 & 0.889 & 0.885 & & \\
\hline Axis 3 & 0.657 & 0.054 & & \\
\hline Axis 4 & -0.124 & -0.105 & & \\
\hline \multicolumn{5}{|c|}{ Medium gradient $(5.1,5.4$ SDU) } \\
\hline Axis 1 & 0.993 & 0.988 & 183.62 & 0.913 \\
\hline Axis 2 & 0.943 & 0.927 & & \\
\hline Axis 3 & 0.499 & 0.328 & & \\
\hline Axis 4 & 0.648 & 0.601 & & \\
\hline \multicolumn{5}{|c|}{ Short gradient $(2.5,2.6 \mathrm{SDU})$} \\
\hline Axis 1 & 0.975 & 0.951 & 19.98 & 0.882 \\
\hline Axis 2 & 0.838 & 0.843 & & \\
\hline Axis 3 & 0.347 & 0.414 & & \\
\hline Axis 4 & 0.568 & 0.278 & & \\
\hline \multicolumn{5}{|c|}{ Short gradient $(2.3,2.4$ SDU $)$} \\
\hline Axis 1 & 0.883 & 0.861 & 7.94 & 0.665 \\
\hline Axis 2 & -0.016 & 0.014 & & \\
\hline Axis 3 & -0.511 & -0.635 & & \\
\hline Axis 4 & 0.257 & 0.300 & & \\
\hline \multicolumn{5}{|c|}{ Short gradient $(1.7,1.8$ SDU $)$} \\
\hline Axis 1 & 0.866 & 0.900 & 4.03 & 0.594 \\
\hline Axis 2 & -0.088 & -0.156 & & \\
\hline Axis 3 & 0.020 & -0.038 & & \\
\hline Axis 4 & -0.011 & -0.021 & & \\
\hline
\end{tabular}

\section{Medium gradient}

To illustrate a medium long gradient (ca 5 SDU), the 75 samples constituting Groups I and II from the first analysis were selected. This data set consists of the shelf stations down to about $200 \mathrm{~m}$ depth. Fig. 2 shows the ordinations based on numerical abundances and biomass values respectively. Compared to the first analyses, the direction of the major gradient (Axis 1 ) is now reversed. The similarity between the 2 ordinations is still high, reflected in a trace value only slightly

Table 3. Modified Jaccard similarity indexes $\left(S_{j}\right)$ for TWINSPAN classification based on numerical vs biomass abundance, for gradients of 5 different lengths

\begin{tabular}{|cccccc|}
\hline Level & Long (9.4 SDU) & Medium (5.4 SDU) & Short (2.6 SDU) & Short (2.4 SDU) & Short (1.8 SDU) \\
\hline 1 & 1 & 1 & 1 & 0.83 & 0.60 \\
2 & 0.94 & 0.90 & 0.85 & 0.45 \\
3 & 0.81 & 0.83 & 0.55 & \\
4 & 0.81 & 0.63 & & \\
5
\end{tabular}


lower than the one for the longer gradient, and is also apparent from the Pearson and Spearman correlation coefficients for the first 2 axes. There is actually a higher correlation between Axes 2 than in the case of the long gradient (Table 2). The impression is still that DCAn results in somewhat more well-defined groups with higher resolution than DCAb, especially as regards the clear distinction between the 2 subgroups of
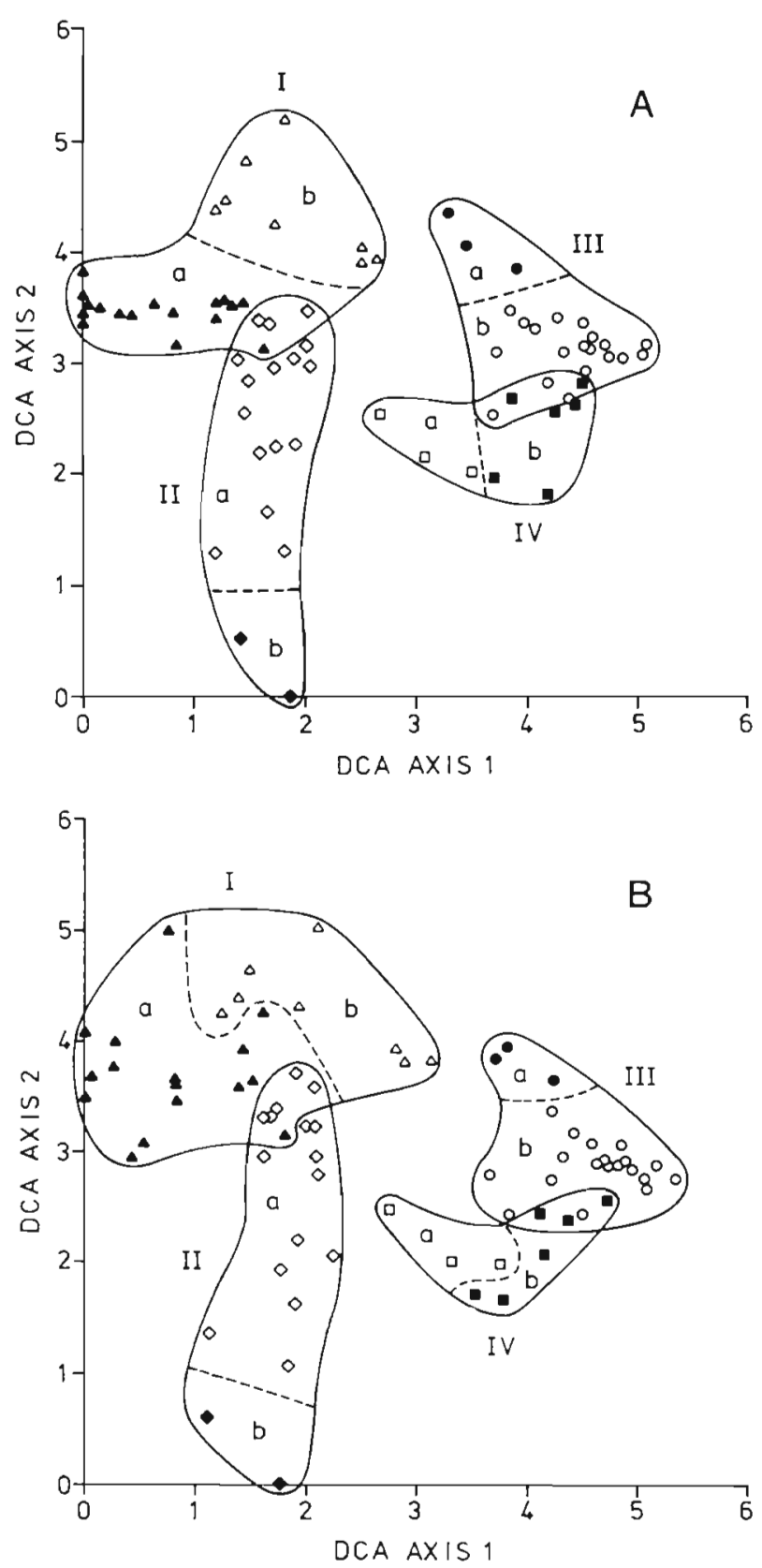

Fig. 2. Detrended correspondence analysis of a subset of 75 stations of the Congo-Gabon material, medium gradient (SDU). Plots based on (A) numerical abundance and (B) biomass. Corresponding groups from TWINSPAN based on presence/ absence data are indicated by different symbols, Roman numerals and lower-case letters
Group I. It is also obvious that the eigenvalues and lengths of gradients for Axis 2 are actually much higher relative to the values for Axis 1 , for the medium than for the long gradient (Tables $1 \& 4$ ).

The similarity between the TWINSPAN classifications for this gradient is perfect at the first division level, becomes slightly lower at the second and third, and drops abruptly at the fourth division level (Table 3 ). Thus only about $60 \%$ of the stations at this level are shared between the 8 clusters of the classification based on numbers and the one based on biomass values.

Table 4. As in Table 1 but for the medium long gradient (75 stations)

\begin{tabular}{|lllll|}
\hline & Axis 1 & Axis 2 & Axis 3 & Axis 4 \\
\hline Eigenvalue & & & & \\
DCAn & 0.746 & 0.455 & 0.266 & 0.183 \\
DCAb & 0.773 & 0.482 & 0.411 & 0.254 \\
DCAp/a & 0.697 & 0.430 & 0.246 & 0.191 \\
& & & & \\
Length of gradient & & & \\
DCAn & 5.1 & 5.2 & 3.4 & 2.8 \\
DCAb & 5.4 & 5.0 & 5.3 & 5.0 \\
DCAp/a & 5.0 & 4.7 & 3.7 & 2.3 \\
\hline
\end{tabular}

\section{Short gradients}

To illustrate a number of 'short' environmental gradients, the 24 stations belonging to Group III in the plots used for medium gradient were selected (Table 5). This data set was then further reduced in 2 steps (Tables 6 \& 7 ), first by removing a group of 3 stations clearly separated from the remaining groups in Fig. 3, and finally a further 5 somewhat scattered stations, to show what happens with data sets representing really short environmental gradients below values of, say, 2 SDU. Figs. 3 , 4 and 5 show the plots resulting from DCAn and DCAb for these data sets, representing ca 2.5, 2.3 and 1.7 SDU respectively. Visual inspection of the plots shows that the similarity is still very high in the case of the longest (24 station 'gradient') of the 3 'short' gradients, while the correspondence falls of steeply between this and the next (21 station 'gradient') plots. This impression is confirmed by the values of the correlation coefficients in Table 2, where the trace for the RDA analysis is still high for the 24 station plots while it falls off to a significantly lower level for the remaining plots. The same result is shown by the correlation between individual axes, for which an apparent discontinuity in the gradual decline of these values is seen between the 24 station values and the ones for the 21 station analysis. This is mainly due to a change in ranking of the axes, as the former Axis 1 becomes Axis 2 after removal of the 3 stations in Groups III and IV in Fig. 3. Thus the correlation between Axes 1 
Table 5. As in Table 1 but for shorter gradient (24 stations)

\begin{tabular}{|lclll|}
\hline & Axis 1 & Axis 2 & Axis 3 & Axis 4 \\
\hline Eigenvalue & & & & \\
DCAn & 0.401 & 0.226 & 0.147 & 0.062 \\
DCAb & 0.365 & 0.257 & 0.143 & 0.095 \\
DCAp/a & 0.389 & 0.221 & 0.148 & 0.100 \\
Length of gradient & & & \\
DCAn & 2.5 & 2.3 & 1.7 & 1.3 \\
DCAb & 2.6 & 2.2 & 2.1 & 1.8 \\
DCAp/a & 2.5 & 2.2 & 1.7 & 1.7 \\
\hline
\end{tabular}

Table 6 . As in Table 1 but for shorter gradient (21 stations)

\begin{tabular}{|lclll|}
\hline & Axis 1 & Axis 2 & Axis 3 & Axis 4 \\
\cline { 1 - 1 } Eigenvalue & & & & \\
DCAn & 0.281 & 0.168 & 0.102 & 0.038 \\
DCAb & 0.311 & 0.181 & 0.117 & 0.086 \\
& & & & \\
Length of gradient & & & \\
DCAn & 2.3 & 1.7 & 1.4 & 1.3 \\
DCAb & 2.4 & 2.0 & 1.7 & 1.5 \\
\hline
\end{tabular}

Table 7. As in Table 6 but for shorter gradient (16 stations)

\begin{tabular}{|lcccc|}
\hline & Axis 1 & Axis 2 & Axis 3 & Axis 4 \\
\hline Eigenvalue & & & & \\
DCAn & 0.230 & 0.172 & 0.109 & 0.039 \\
DCAb & 0.245 & 0.170 & 0.067 & 0.031 \\
& & & & \\
Length of gradient & & & \\
DCAn & 1.7 & 1.7 & 1.5 & 1.2 \\
DCAb & 1.8 & 1.8 & 1.5 & 1.2 \\
\hline
\end{tabular}

falls from 0.98 to 0.88 , while that for Axes 2 falls from 0.84 to zero, for this small drop in length of environmental gradient (from 2.5 and 2.6 in the former case to 2.3 and 2.4 in the latter). While it is still fairly easy to identify corresponding groups in the 2 DCA plots for the 24 station 'gradient', such an identification is only possible with some difficulty for 2 or 3 of the subgroups in the plots for the 21 station 'gradient', and none of the groups in the 16 station 'gradient' plots can be so identified. The dramatic drop in the $F$-ratios from the medium to the short gradients, as shown in Table 2, is less easily interpreted. The comparisons between the TWINSPAN classifications (Table 3) show that while, for the 24 station classification, the correspondence is still excellent to good for the first 2 levels (down to 0.55 at the third level, however), only for the first level an acceptable correspondence remains for the 21 station classification, and very little, if any, similarity is evident for the 2 levels shown for the 16 station classification.
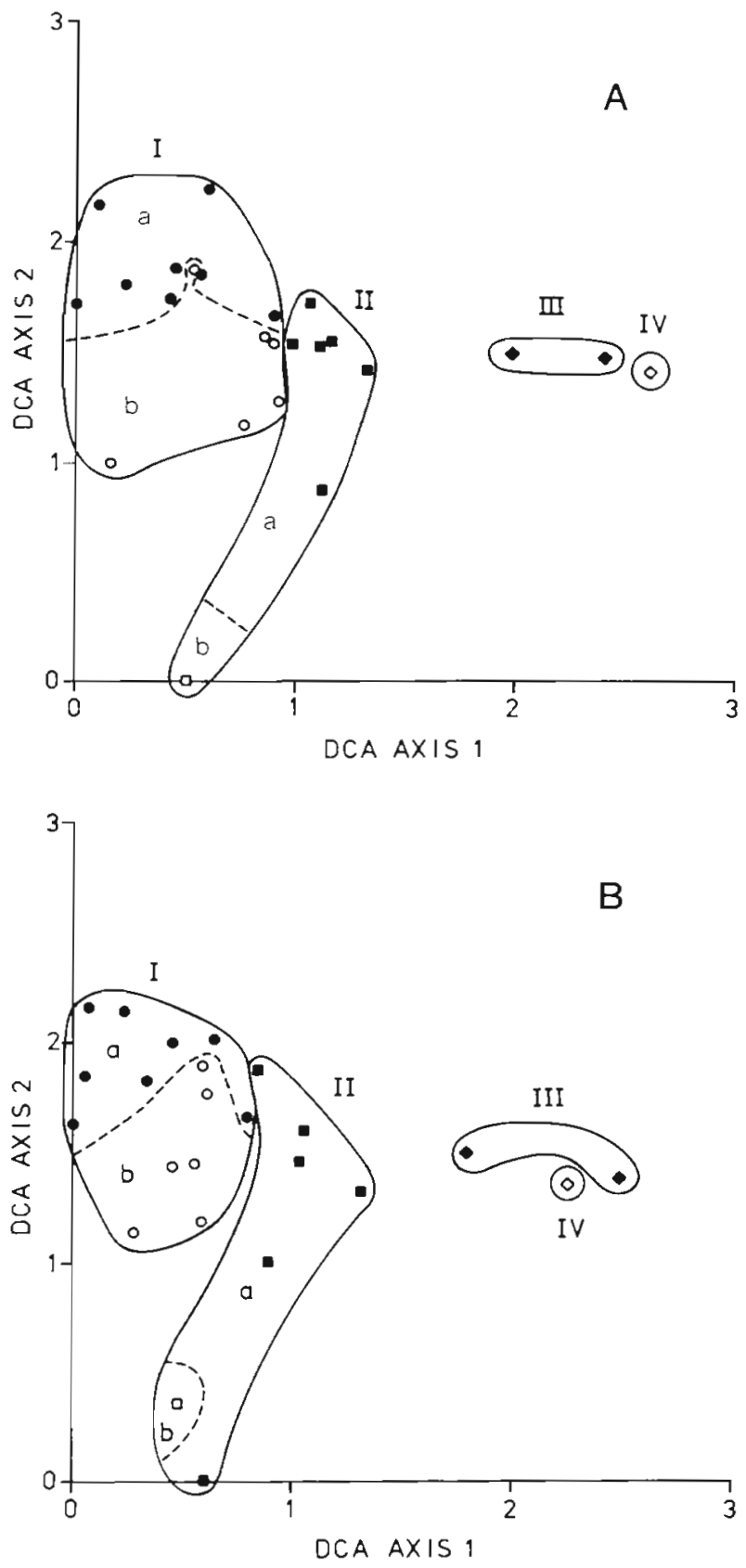

Fig. 3. Detrended correspondence analysis of a subset of 24 stations of the Congo-Gabon material, short gradient (SDU). Plots based on (A) numerical abundance and (B) biomass. Corresponding groups from TWINSPAN based on presence/ absence data are indicated by different symbols, Roman numerals and lower-case letters

\section{DISCUSSION}

When discussing the relative merits of various measures of abundance (or 'importance value') for multivariate analyses of animal communities, it is important to take into account the purpose of the investigation, as 

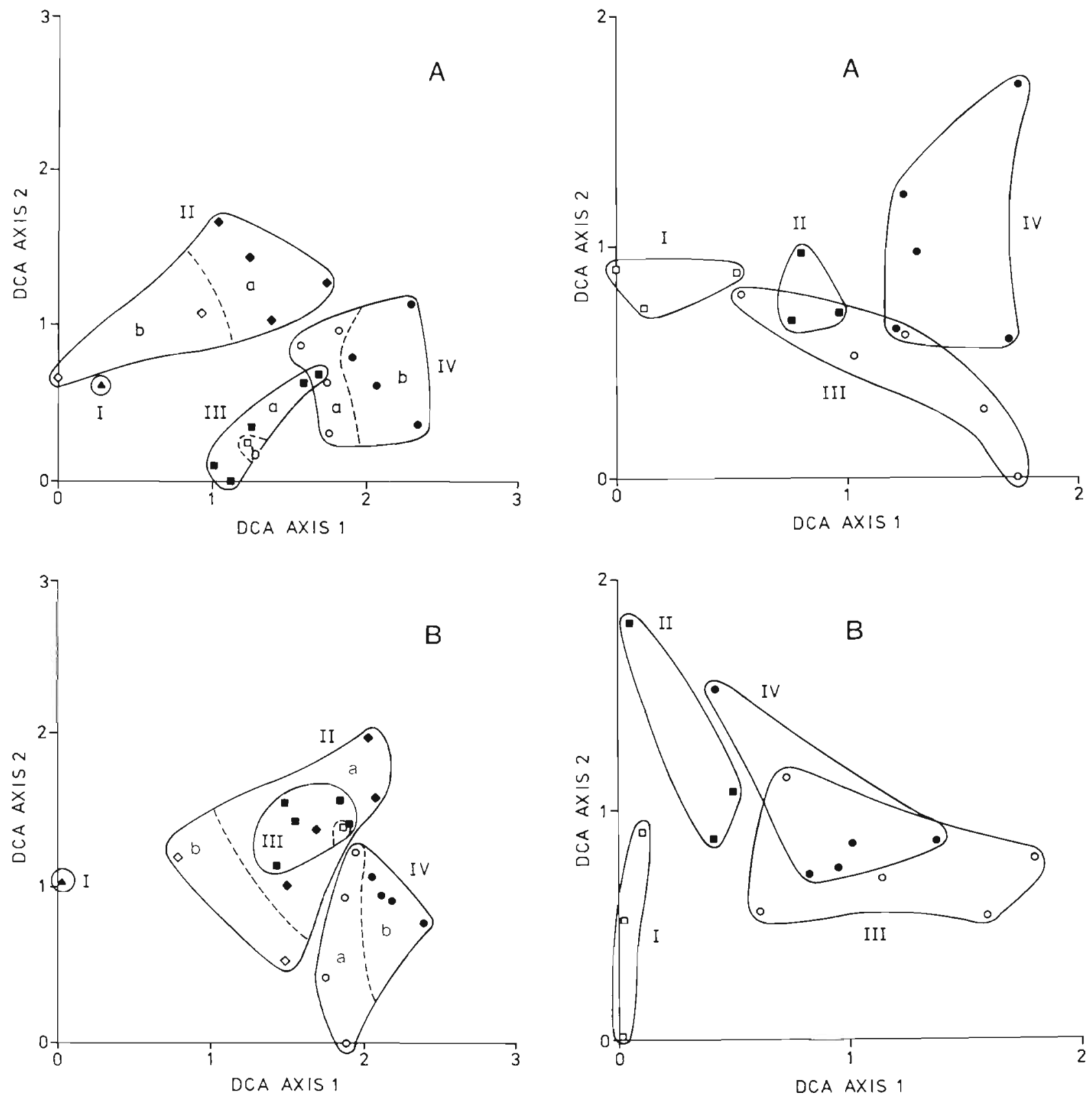

Fig. 4. Detrended correspondence analysis of a subset of 21 stations of the Congo-Gabon material, 'shorter' gradient (SDU). Plots based on (A) numerical abundance and (B) biomass. Corresponding groups from TWINSPAN based on presence/absence data are indicated by different symbols, Roman numerals and lower-case letters

Fig. 5. Detrended correspondence analysis of a subset of 16 stations of the Congo-Gabon material, shortest gradient (SDU). Plots based on (A) numerical abundance and (B) biomass. Corresponding groups from TWINSPAN based on presence/absence data are indicated by different symbols. Roman numerals and lower-case letters

well as the nature of the data. Thus, in a purely exploratory survey, when the sole purpose is to establish coarse 'associations' of organisms living in a particular geographical region, it is probably unnecessary to proceed beyond presence/absence. The only condition is that the samples (or catches) are large enough for the

stray singletons to be unimportant. In cases when finer detail is wanted, and especially when correlations with environmental or biotic data are to be established, the choice between numbers (or numerical density) and biomass could be critical. Of course, if all the organisms of interest are more or less of the same size, the 
distinction will become academic, and can safely be ignored. In comparing fish communities, especially if based on trawl catches in which a wide spectrum of size categories is represented, the choice could be critical, however. The problem of which value (either numbers or biomass) should be representative of species abundance in community studies rises just because there is usually a wide variation in individual weight among species and within the same species at different ages.

It appears from our study that the choice between these 2 abundance values is not critical in the case of community analyses based on methods related to Correspondence Analysis, including TWINSPAN. This is true for data sets covering long gradients, while a clear decreasing correspondence is shown when analysing short gradients.

There is a high degree of correspondence between the plots based on numbers and biomass for the 3 longest environmental gradients, while the correspondence deteriorates rapidly somewhere between a gradient of 2.6 and 2.3 SDU (Tables $2 \& 3$ ). Thus, while much valuable information is to be gleaned from a juxtaposition of the 2 categories of plots, for most investigations covering long environmental gradients (more than about 2.5 SDU), biomass and numbers seem to be about equally suitable as abundance measures for exploratory studies of fish communities. The increase in correlation between Axes 2 from the long to the medium DCA's (Table 2) may be because the influence of the outlier station in the long gradient biomass plot is much reduced in the corresponding medium gradient plot.

The reason for the similarity in the results of ordinations/classifications using different types of abundance data can be explained by the nature of CA and related methods. The double standardization implied in the algorithm (to species total for a species and to site total for a site) reduces the quantitative aspect of the data. For this reason, and particularly if the data have been log-transformed, the differences between the use of numbers or biomass will be minimal. This is obviously true only when the data analysed cover long gradients, i.e. where there is a clear succession of species along a gradient, in the area covered. In the case of short gradients, as for example in the extreme case where all species are common to all sites, the quantitative aspects will become more important and the differences in the results based on numerical abundance and biomass will become more conspicuous.

The 2 different aspects implied in abundance data, viz. the qualitative aspect (presence or absence of the species) and the quantitative aspect i.e. regarding differences in abundance when the species is present, are thus differently stressed depending on the multi- variate analysis techniques used and/or the length of the ecological gradient covered by the data set.

The generality of these conclusions may of course be challenged on the grounds that this particular data set is incapable of illustrating the many facets of natural fish communities representing various environmental/ biogeographical gradients. A more ideal approach would probably be to analyse a series of simulated fish communities, in which the environmental gradient is varied over a broad spectrum, and various levels of noise are introduced. With the fragmentary knowledge of the assemblage rules for fish communities, this possibility is not very realistic at the moment, and we could not see any realistic alternative to the present investigation for our purpose. Another possible objection to the generality of the results is that other multivariate methodologies might be more sensitive to the choice of abundance measures than DCA and TWINSPAN, and we do not of course claim that our conclusions could be extrapolated to other such methods.

An investigation of this type is incapable of settling the question of which abundance measure is 'best', whenever the correspondence between 2 alternatives is small or nonexistent. When, as in this case, the correspondence falls from excellent or good to dismal with a shrinking environmental gradient, it may be because there is really no natural pattern in the data, and that any attempt at grouping would be equally meaningless. A possible explanation for lack of meaningful patterns in material of this kind is the presence of sampling 'noise', which would be more serious the shorter the gradient sampled. The potential effects of such sampling noise cannot be disregarded in our chosen example. A trawl of the kind used in this investigation is not at all immune to such criticism, and both the way it works, as well as seasonal and diurnal variations in the swimming behaviour of the animals sampled, will influence the reproducibility of the catches. Thus it is perhaps best to restrict this kind of investigation to environmental gradients longer than ca 2.5 SDU. In the spirit of Gordon (1982, p. 134), it might be wise only to accept as 'good' groups those clearly identifiable from analyses of both numerical abundance and biomass.

Acknowledgements. We thank H. J. B. Birks for suggesting the use of RDA for comparing DCA analyses and for reading an early version of the manuscript, and $\mathrm{E}$. Holm for drawing the figures.

\section{LITERATURE CITED}

Bechtel, T J., Copeland, B. J (1970). Fish species diversity indexes as indicators of pollution in Galveston Bay, Texas. Contr. mar. Sci. 15: 101-132

Bennett, B. A. (1989). The fish community of a moderately exposed beach on the southwestern Cape coast of South 
Africa and an assessment of this habitat as a nursery for juvenile fish. Estuar. coast. mar. Sci. 28: 293-305

Bergstad, O. A. (1991). Distribution and trophic ecology of some gadoid fish of the Norwegian deep. 2. Food-web linkages and comparisons of diets and distributions. Sarsia 75: 315-325

Bianchi, G. (1991). Demersal assemblages of the continental shelf and slope edge between the Gulf of Tehuantepec (Mexico) and the Gulf of Papagayo (Costa Rica). Mar. Ecol. Prog. Ser. 73: 121-140

Bianchi, G. (1992a). Demersal assemblages of the continental shelf and upper slope of Angola. Mar. Ecol. Prog. Ser. 81. $101-120$

Bianchi, G. (1992b). Study of the demersal assemblages of the continental shelf and upper slope of Congo and Gabon, based on the trawl surveys of the RV 'Dr Fridtjof Nansen' Mar. Ecol. Prog. Ser. 85: 9-23

Chavez, E. A. (1979). Análisis de la comunidad de una laguna costerade la costa suroccidental de México. An. Cent. Cienc. Mar Limnol., Univ. nac. Autónoma México 6(2): $15-44$

Colvocoresses, J. A., Musick, J. A. (1984). Species associations and community composition of Middle Atlantic Bight continental shelf demersal fishes. Fish. Bull. U.S. 82: $295-313$

Costa, M. J. (1986). Les poissons de l'estuaire du Tage. Cybium 10:57-75

Day, D. S., Pearcy, W. G. (1968). Species associations of benthic fishes on the continental shelf and slope off Oregon. J. Fish. Res. Bd Can. 25: 2665-2675

Fager, E. W., Longhurst, A. R. (1968). Recurrent group analysis of species assemblages of demersal fish in the Gulf of Guinea. J. Fish. Res. Bd Can. 25: 1405-1421

Gabriel, W. L., Tyler, A. V. (1980). Preliminary analysis of Pacific Coast demersal fish assemblages. Mar. Fish. Rev. 42(3-4): $83-88$

Gordon, A. D. (1982). Classification. Methods for the exploratory analysis of multivariate data. Chapman and Hall, London

Haedrich, R. L., Rowe, G. T., Polloni, P. T (1975). Zonation and faunal composition of epibenthic populations on the continental slope south of New England. J. mar. Res. 33: 191-212

Haedrich, R. L., Rowe, G. T., Polloni, P. T. (1980). The megabenthic fauna in the deep sea south of New England, USA. Mar. Biol. 57: 165-179

Hill, M. O. (1979). TWINSPAN - a FORTRAN program for arranging multivariate data in an ordered two-way table by classification of individuals and attributes. Cornell University Ithaca, New York

Hill, M. O., Gauch, H. G. (1980). Detrended correspondence analysis, an improved ordination technique. Vegetatio 42 : $47-58$

Jackson, D. A., Somers, K. M. (1991). Putting things in order: the ups and downs of Detrended Correspondence Analysis. Am. Nat. 137. 704-712
Jongman, R. H. G., ter Braak, C. J. F., van Tongeren, O. F. R. (1987). Data analysis in community and landscape ecology. Pudoc Wageningen, The Netherlands

Lleonart, J., Roel, B. A. (1984). Análisis de las comunidades de peces y crustáceos demersales de la costa de Namibia (Atlántico Sudoriental). Investigación pesq. 48: 187-206

Mahon, R., Smith, R. W. (1989). Demersal fish assemblages on the Scotian shelf, northwest Atlantic: spatial distribution and persistence. Can. J. Fish. Aquat. Sci. 46: 134-152

Markle, D. F., Musick, J. A. (1974). Benthic-slope fishes found at $900 \mathrm{~m}$ depth along a transect in the western North Atlantic ocean. Mar. Biol. 26: 225-233

Mas-Riera, J., Lombarte, A., Gordoa, A., Macpherson, E. (1990). Influence of Benguela upwelling on the structure of demersal fish populations off Namibia. Mar. Biol. 104: $175-182$

McManus, J. W. (1985). Descriptive community dynamics: background and an application to tropical fisheries management. Ph.D. thesis, University of Rhode Island, Providence

Overholtz, W. J., Tyler, A. V. (1984). Long-term responses of the demersal fish assemblages of Georges Bank. Fish. Bull. U.S. 83: 507-520

Rainer, S. F. (1984). Temporal changes in a demersal fish and cephalopod community of an unexploited coastal area in northern Australia. Aust. J. mar. Freshwat. Res. 35: $747-768$

Roel, B. A. (1987). Demersal communities off the west coast of South Africa. In: Payne, A. I. L., Gulland, J. A., Brink, K. H. (eds.) The Benguela and comparable ecosystems. S. Afr. J. mar. Sci. 5: 575-584

ter Braak, C. J. F. (1990). CANOCO - a FORTRAN program for canonical community ordination by (partial) (detrended) (canonical) correspondence analysis, principal component analysis and redundancy analysis (version 3.10). ITI-TNO, Wageningen

Vargas Maldonado, I., Yanéz-Arancibia, A., Amezuca Linares, F. (1981). Ecología y estructura de las comunidades de peces en áreas de Rhizophora mangle y Thalassia testudinum de la isla del Carmen, Laguna de Terminos, sur del Golfo de México. An. Inst. Cienc. del Mar y Limnol, Univ. nac. Autónoma México 8(1): 241-266

Warburton, K. (1978). Community structure, abundance and diversity of fish in a coastal lagoon system. Estuar. coast. mar. Sci. 7: 497-519

Wilhm, J. L. (1968). Use of biomass units in Shannon's formula. Ecology 49: 153-156

Yañéz-Arancibia, A., Amezcua Linares, F., Day, J. W. Jr (1980). Fish community structure and function in Terminos Lagoon, a tropical estuary in the southern Gulf of Mexico. In: Kennedy, V. (ed.) Estuarine perspectives. Academic Press, New York, p. 465-482

Yañéz-Arancibia, A., Sanchez-Gil, P., Tapia Garcia, M., Garcia-Abad, M. de la C. (1985). Ecology, community structure and evaluation of tropical demersal fishes in the southern Gulf of Mexico. Cah. Biol. Mar. 26: 137-163

Manuscript first received: March 4, 1992 Revised version accepted: June 22, 1992 